to treat many other disorders (because PEG prolongs bioavailability), PEG immunogenicity should be investigated further.

Katherine Sole

Original article Ganson NJ et al. (2006) Control of hyperuricemia in subjects with refractory gout, and induction of antibody against poly(ethylene) glycol (PEG), in a phase I trial of subcutaneous PEGylated urate oxidase. Arthritis Res Ther 8: R12

\section{Pegsunercept is a safe and efficacious treatment for rheumatoid arthritis}

Preclinical studies have indicated that pegsunercept, a polyethylene-glycol-modified soluble tumor-necrosis-factor receptor type 1, is effective in treating rheumatoid arthritis (RA). Pegsunercept has also been shown to be well tolerated in a clinical trial. Investigators in the US have conducted a prospective, doubleblind, randomized, placebo-controlled, multicenter, phase II trial, assessing the safety and efficacy of this treatment for RA.

In total, 195 patients (154 female) were allocated to receive weekly injections of either placebo $(n=62)$ or pegsunercept $400 \mu \mathrm{g} / \mathrm{kg}(n=67)$ or $800 \mu \mathrm{g} / \mathrm{kg}(n=66)$, for 12 weeks. Efficacy was defined as the proportion of patients who showed an ACR20 response at week 12, compared with their baseline score. Clinical efficacy was defined as a difference of $25 \%$ in ACR20 scores between the placebo group and either of the active-treatment groups.

The response rate was statistically (but not clinically) higher in the $800 \mu \mathrm{g} / \mathrm{kg}$ group than in the placebo group ( $45 \%$ versus $26 \%, P=0.02$ ). No significant difference was found between the response rates of the $400 \mu \mathrm{g} / \mathrm{kg}$ group and the placebo group. The incidence of adverse events was similar across all three groups, and most adverse events were mild-to-moderate in severity. Two patients experienced serious adverse events; neither event was related to use of pegsunercept.

Pegsunercept seems to be safe and efficacious at an $800 \mu \mathrm{g} / \mathrm{kg}$ dose; however, further dose-finding studies are warranted, as higher dosages or more frequent dosing might improve response rates even more.

Katherine Sole

Original article Furst DE et al. (2005) A phase 2 dosefinding study of PEGylated recombinant methionyl human soluble tumor necrosis factor type I in patients with rheumatoid arthritis. J Rheumatol 32: 2303-2310

\section{More support for MRI-based screening of joint damage in rheumatoid arthritis}

Joint damage in rheumatoid arthritis $(R A)$ is conventionally assessed radiographically, but MRI can provide greater detail without the adverse effects of ionizing radiation. RAMRIS, a semiquantative MRI-based system for scoring joint damage in the hand and wrist, was developed by OMERACT as an outcome measure in clinical trials, but earlier studies indicated that it was less reliable for monitoring change. A new Norwegian study has found, however, that RAMRIS showed very good intrareader and inter-reader reliability for change, when digital images were scored by trained, calibrated readers.

A key feature of the study was that the four readers met for a day's training, 1 month before the study, to review scoring methods and train the least-experienced readers. They independently scored baseline and 1-year follow-up MRI scans of the wrist joints from 10 patients with RA who showed radiographic progression; 4 with early and 6 with established disease. Images were scored for synovitis, and each wrist bone was assessed for erosion and edema. Intrareader and inter-reader reliability was high for all components of the status and change scores (average-measure intraclass correlation coefficients all $\geq 0.94$ ). The reliability of the RAMRIS scores in this study are comparable to those published for radiographic erosion-scoring methods.

The authors suggest that RAMRIS is suitable for longitudinal observational studies, as well as clinical practice, and randomized controlled trials.

Caroline Barranco

Original article Haavardsholm EA et al. (2005) Reliability and sensitivity to change of the OMERACT rheumatoid arthritis magnetic resonance imaging score in a multireader, longitudinal setting. Athritis Rheum 52: 3860-3867

\section{Antiferritin antibodies are potential biomarkers for rheumatoid arthritis}

Previous studies demonstrated that antiferritin antibodies are associated with radiographic damage in patients with rheumatoid arthritis (RA). Autoantibodies could be useful in defining clinically relevant subgroups of patients with RA

\section{GLOSSARY \\ ACR20 \\ $\geq 20 \%$ improvement in rheumatoid arthritis signs and symptoms (swollen and tender joint count, acute-phase reactant level, physician and patient response assessment and health assessment questionnaire) according to American College of Rheumatology criteria}

RHEUMATOID ARTHRITIS MRI SCORE (RAMRIS) MRI-based system for scoring joint damage and inflammation in clinical trials; developed and endorsed by the Outcome Measures in Rheumatology MRI group

OUTCOME MEASURES IN RHEUMATOLOGY (OMERACT)

An informal international network that holds biennial meetings, to develop consensus guidelines for outcome measurement in rheumatic diseases 